\title{
Levosimendan improves cardiac function and survival in rats with angiotensin II-induced hypertensive heart failure
}

\author{
Agnieszka Biala ${ }^{1}$, Essi Martonen ${ }^{1}$, Petri Kaheinen ${ }^{1}$, Jouko Levijoki ${ }^{2}$, Piet Finckenberg${ }^{1}$, Saara Merasto ${ }^{1}$, \\ Marjut Louhelainen ${ }^{1}$, Dominik N Muller ${ }^{3}$, Friedrich C Luft ${ }^{3}$ and Eero Mervaala ${ }^{1}$
}

Calcium-sensitizing agents improve cardiac function in acute heart failure; however, their long-term effects on cardiovascular mortality are unknown. We tested the hypothesis that levosimendan, an inodilator that acts through calcium sensitization, opening of ATP-dependent potassium channels and phosphodiesterase III inhibition, improves cardiac function and survival in double transgenic rats harboring human renin and angiotensinogen genes (dTGRs), a model of angiotensin II (Ang II)-induced hypertensive heart failure. Levosimendan $\left(1 \mathrm{mg} \mathrm{kg}^{-1}\right)$ was administered orally to 4-week-old dTGRs and normotensive Sprague-Dawley rats for 4 weeks. Untreated dTGRs developed severe hypertension, cardiac hypertrophy, heart failure with impaired diastolic relaxation, and exhibited a high mortality rate at the age of 8 weeks. Levosimendan did not decrease blood pressure and did not prevent cardiac hypertrophy. However, levosimendan improved systolic function, decreased cardiac atrial natriuretic peptide mRNA expression, ameliorated Ang II-induced cardiac damage and decreased mortality. Levosimendan did not correct Ang II-induced diastolic dysfunction and did not influence heart rate. In a separate survival study, levosimendan increased dTGR survival by $58 \%$ and median survival time by $27 \%(P=0.004)$. Our findings suggest that levosimendan ameliorates Ang II-induced hypertensive heart failure and reduces mortality. The results also support the notion that the effects of levosimendan in dTGRs are mediated by blood pressure-independent mechanisms and include improved systolic function and amelioration of Ang II-induced coronary and cardiomyocyte damage.

Hypertension Research (2010) 33, 1004-1011; doi:10.1038/hr.2010.123; published online 2 September 2010

Keywords: angiotensin II; calcium sensitizers; heart failure; hypertrophy

\section{INTRODUCTION}

Defects in cardiac excitation-contraction coupling have been identified in both human and experimental models of heart failure characterized as prolonged calcium transient with elevated enddiastolic and decreased systolic intracellular calcium concentrations and prolonged relaxation phase. ${ }^{1,2}$ Excitation-contraction coupling is usually classified into three processes, namely 'upstream mechanisms,' such as $\mathrm{Ca}^{2+}$ mobilization, 'central mechanisms,' such as $\mathrm{Ca}^{2+}$ binding to troponin $\mathrm{C}$ and 'downstream mechanisms', including thin filament regulation and cross-bridge cycling, as reviewed elsewhere. ${ }^{3}$ Classical inotropic agents such as $\beta 1$-adrenergic agonists, phosphodiesterase III (PDE-III) inhibitors and digitalis function primarily through upstream mechanisms. ${ }^{3}$ Although these agents improve symptoms and hemodynamics by increasing intracellular $\mathrm{Ca}^{2+}$ levels, they increase myocardial oxygen demand, exert arrhythmogenic effects and worsen both short- and long-term mortality. ${ }^{3-5}$ Calcium sensitizers are novel drugs acting by combination of the upstream and central/downstream mechanisms. ${ }^{3,5-7}$ Calcium sensitizers seem advan- tageous for treating acute decompensated heart failure and acute lowoutput heart failure, compared with clinically available inotropes, which act primarily by increasing intracellular calcium mobilization in cardiac myocytes. ${ }^{5-7}$ Although evidence supports the idea that $\mathrm{Ca}^{2+}$ sensitivity is decreased during acute cardiac hypoxia or ischemia, ${ }^{8,9}$ data from subacute and chronic heart failure experiments showed that $\mathrm{Ca}^{2+}$ sensitivity may even be increased because of a decrease in troponin I phosphorylation caused by the downregulation of $\beta 1$-adrenoceptor-mediated signaling. ${ }^{3,10}$ Therefore, the role of calcium sensitizers in the treatment of chronic heart failure remains unclear.

Levosimendan binds to the calcium-saturated N-terminal domain of troponin $\mathrm{C}$ in the cardiac muscle and stabilizes the troponin molecule, with subsequent prolongation of its effect on contractile proteins. ${ }^{11-13}$ Besides increasing the strength of cardiac contractions, levosimendan exerts vasodilatory effects by opening sarcolemmal and mitochondrial ATP-sensitive potassium channels. ${ }^{14}$ Levosimendan also potently inhibits PDE-III in vitro. ${ }^{15,16}$ However, the clinical relevance of its PDE-III inhibitory action still remains unclear.

${ }^{1}$ Institute of Biomedioine, University of Helsinki, Helsinki, Finland; ${ }^{2}$ Orion Pharma, Espoo, Finland and ${ }^{3}$ Max Delbrück Center, Experimental and Clinical Research Center, Berlin, Germany

Correspondence: Dr E Mervaala, Institute of Biomedicine, PO Box 63, FI-00014 University of Helsinki, Helsinki, Finland.

E-mail: eero.mervaala@helsinki.fi

Received 18 March 2010; revised and accepted 11 May 2010; published online 2 September 2010 
We showed earlier that levosimendan improved survival and ameliorated hypertension-induced cardiac remodeling in Dahl salt-sensitive rats ${ }^{17}$ and prevented postinfarct heart failure in spontaneously diabetic Goto-Kakizaki rats. ${ }^{18}$ Furthermore, Nieminen et al. ${ }^{19}$ showed that patients with severe chronic heart failure showed improved quality of life and reduced plasma brain natriuretic peptide levels, although their cardiovascular mortality did not decrease. We now investigated the effects of levosimendan in a novel, high-angiotensin (Ang) II, hypertensive heart failure model. We reasoned that the renin-angiotensin system is pivotal to the heart failure process as targeted drug interventions of this system are so successful. We used a double transgenic rat model harboring human renin and angiotensinogen genes (dTGRs). ${ }^{20-23}$

\section{METHODS}

\section{Experimental animals, blood pressure measurement and sample} preparation

We used 75 4-week-old male double transgenic rats (dTGRs) (Biotechnology and Animal Breeding Division, Füllingsdorf, Switzerland) and 20 age-matched normotensive Sprague-Dawley (SD) rats (Charles River, Schutzfeld, Germany) as described elsewhere. ${ }^{22}$ The protocols were approved by the Animal Experimentation Committee of the University of Helsinki, Finland, and by the Provincial State Office of Southern Finland (approval number STU 1187 A), the standards of which correspond to those of the American Physiological Society. The rats had free access to chow (SDS Special Diet Services, Witham, Essex, UK) and drinking water. Both dTGRs and normotensive SD control rats were divided into four groups to receive the following diet and drug regimens for 4 weeks: (1) dTGR controls $(n=20)$; (2) dTGR+levosimendan $(n=15)$; (3) SD controls $(n=10)$; (4) SD+levosimendan $(n=10)$. The length of the followup period was based on our previous studies showing pronounced cardiovascular mortality already at the age of 8 weeks. ${ }^{20-23}$ However, in a separate survival study, the influence of lifelong oral levosimendan treatment on cardiovascular mortality in dTGRs was examined (dTGR controls, $n=20$; dTGR treated with levosimendan, $n=20$ ). Systolic blood pressure was measured weekly from week 6 to week 8 using a tail-cuff blood pressure analyzer (Apollo2AB Blood Pressure Analyzer, Model 179-2AB, IITC Life Science, Woodland Hills, CA, USA). At the end of the follow-up period, urine samples were collected over a 24 -h period in metabolic cages for albumin measurement. Urine volumes and water intakes were measured gravimetrically. At the age of 8 weeks, rats were rendered unconscious with $\mathrm{CO}_{2} / \mathrm{O}_{2}(95 \% / 5 \%$, AGA, Riihimäki, Finland) in $10 \mathrm{~s}$, and then decapitated (national legislation 20.1.2006/62). Blood samples were collected for biochemical measurements using EDTA (ethylenediamine tetraacetic acid) as an anticoagulant. The hearts and kidneys were excised, washed with ice-cold saline, blotted dry and weighed. Tissue samples for histology were fixated in $10 \%$ formalin and processed to paraffin with routine methodology. For protein and gene expression studies, cardiac samples were snap frozen in liquid nitrogen and stored at $-80^{\circ} \mathrm{C}$ until assayed. Levosimendan (a kind gift from Orion Pharma, Espoo, Finland) was administered orally through drinking fluid to produce an approximate daily dosage of $1 \mathrm{mg} \mathrm{kg}^{-1}$ as in our previous rat experiments. ${ }^{17,18}$ Fresh levosimendan solutions were prepared daily. Owing to the dipsogenic effect of Ang $\mathrm{II}^{24,25}$ the water intake in dTGRs is approximately three-fold higher compared with SD rats. We therefore adjusted the levosimendan concentration in drinking fluid at threefold higher concentration for $\mathrm{SD}$ rats (levosimendan concentration in drinking fluid $3 \mathrm{mgl}^{-1}$ for dTGRs and $10 \mathrm{mgl}^{-1}$ for SD rats).

\section{Echocardiography}

Transthoracic echocardiography (Toshiba Ultrasound, Tokyo, Japan) was performed on all rats under isoflurane anesthesia (AGA) in a blinded manner by the same technician during the last study week as described previously.18 Parameters required for the calculation of cardiac function and cardiac dimensions were measured from three systole-diastole cycles. A short-axis view of the left ventricle (LV) at the level of the papillary muscles was obtained by a two-dimensional imaging method (Gibson method), using a $15-\mathrm{MHz}$ linear transducer. Two dimensionally guided M-mode recording through the anterior and posterior walls of the LV was used to measure both LVESD (LV end-systolic dimension) and LVEDD (LV end-diastolic dimension). In addition, interventricular septum and posterior wall thickness were measured. LV fractional shortening (FS) and ejection fraction (EF) were calculated from the M-mode LV dimensions using the following equations:

$$
\begin{aligned}
& \mathrm{FS}(\%)=\{(\mathrm{LVEDD}-\mathrm{LVESD}) / \mathrm{LVEDD}\} \times 100 \\
& \mathrm{EF}=\mathrm{SV} / \mathrm{EDV} \\
& \mathrm{SV}=\mathrm{EDV}-\mathrm{ESV} \\
& \mathrm{EDV}=0.52 \times(0.98 \times(\mathrm{LVIDD} / 10)+5.90) \times(\mathrm{LVIDD} / 10)^{2} \\
& \mathrm{ESV}=0.52 \times(1.14 \times(\mathrm{LVIDS} / 10)+4.18) \times(\mathrm{LVIDS} / 10)^{2}
\end{aligned}
$$

LVEDD $=$ diameter of the short-axis LV in end diastole. LVEDS=diameter of the short-axis LV in end systole. We assessed diastolic dysfunction by measuring the isovolumic relaxation time using color Doppler imaging. The isovolumic relaxation time was measured as the interval between the aortic closure click and the start of mitral flow.

\section{Tissue morphology and cardiomyocyte cross-sectional area}

Tissue morphology was evaluated from hematoxylin and eosin-stained cardiac and renal sections in a blinded manner. The severity of observed lesions was graded with numerical values denoting the degree of damage at the whole tissue level. The following system of severity grading was used: ( 0 , no abnormalities detected) 1 , minimal; 2 , mild; 3 , moderate; 4 , marked; or 5 , severe. ${ }^{26}$ Conventional light microscopy at $\times 400$ magnification was used to determine cardiomyocyte cross-sectional area. In all, 15-17 random fields were studied, and in each field, the cell borders were measured from myocytes cut in the short axis with a visible nucleus. An average of 50 cardiomyocytes per animal was studied from each animal in the group. The cross-sectional area was evaluated in a blinded manner and analyzed using the ISlimaging software (Image Solutions, Whippany, NJ, USA). ${ }^{17,18}$

\section{Cardiac mRNA expression analysis by quantitative real-time reverse transcriptase-PCR}

Quantitative real-time reverse transcriptase-PCR was performed using the LightCycler instrument (Roche Diagnostics, Neuilly sur Seine, France) for detection of atrial natriuretic peptide (ANP), SERCA2, NCX-1, $\alpha$-MHC, $\beta$-MHC, MCP-1, Bax, PGC- $1 \alpha$ and ribosomal $18 \mathrm{~S}$ mRNA as described elsewhere. ${ }^{18,27}$ Briefly, total RNA from the rat hearts were collected using Trizol (Gibco, Invitrogen, Carlsbad, CA, USA), treated with DNAse 1 (deoxyribonuclease 1, Sigma Chemicals, St Louis, MO, USA) and reverse transcribed to cDNA by reverse transcription enzyme (Im-Prom-II reverse transcription system, Promega, Madison, WI, USA). A volume of $1 \mu$ l of cDNA was subjected to quantitative real-time PCR for detection of ANP, $\alpha$-MHC, $\beta$-MHC, MCP- 1 , Bax, PGC- $1 \alpha$ and ribosomal $18 S$ mRNA. The following primers were used: ANP forward: CCGATAGATTCTGCCCTCTTGAA, reverse: CCCGAAGCAGC TTGATCTTC; $\alpha$-MHC forward: CTGAAAACGGCAAGACGGT, reverse: ACTT ATAGGGGTTGACGGTG; $\beta$-MHC forward: GCCCGGCATGATTGCG, reverse: TGGCGTCCGTCTCATACT; MCP-1 forward: GCAGGTCTCTGTC ACGCTTCT, reverse: GGCTGAGACAGCACGTGGAT; Bax forward: CGGC GAATTGGAGAGATG, reverse: GGTCCCGAAGTAGGAG; PGC- $1 \alpha$ forward: GGTCCCCAGGCAGTAG, reverse: CTCCATCATCCCGCAG, $18 \mathrm{~S}$ forward: CATCCAAGGAAGGCAGCAG, reverse: TTTTCGTCACTACCTCCCCG. The samples were amplified using FastStart DNA Master SYBR Green 1 (Roche Diagnostics) according to the protocol of the manufacturer. The quantities of the PCR products were quantified with an external standard curve amplified from purified PCR product.

\section{Cardiac SERCA2a and NCX expressions by western blot}

Cardiac samples from the LV were electrophoretically separated by $8 \%$ SDS-PAGE $(20 \mu \mathrm{g}$ total protein of the whole-cell lysate per lane). Each lane corresponded to one rat and all four groups were run on one gel. Proteins were transferred onto a polyvinylidene fluoride membrane (Immobilon-P, Millipore, 
Bedford, MA, USA) and blocked in 5\% non-fat milk-TBS-0.01\% Tween-20 buffer. The membranes were probed with the following primary antibodies; anti-Serca2a, 1/1000 (Abcam, Cambridge, Cambridgeshire, UK) and anti-NCX, 1/5000 (Alpha Diagnostic, San Antonio, TX, USA). Tubulin was used as the loading control (anti- $\alpha$ tubulin, 1/2000; Abcam). Horseradish peroxidaseconjugated anti-rabbit secondary antibody (Chemicon, Temecula, CA, USA) was subjected to enhanced chemiluminescence solution (ECLplus, Amersham Biosciences, Buckinghamshire, UK). We quantified the relative protein expression in separate samples from the membranes using Fluorescent Image Analyzer (Fujifilm, Tokyo, Japan). The measurements were repeated three times, and the data were presented as means \pm s.e.m. of these experiments.

\section{Biochemical determinations and statistics}

Urinary albumin was measured by enzyme-linked immunosorbent assay using rat albumin as a standard (Calltrend, Luckenwalde, Germany). Creatinine and electrolytes from the plasma and urine as well as plasma lipids and liver enzymes were measured by routine techniques. Plasma samples were analyzed for levosimendan and OR-1896 by liquid chromatography-tandem mass spectrometry. Data are presented as mean \pm s.e.m. Statistically significant differences in mean values were tested by ANOVA (analysis of variance) and Newman-Keul's post hoc test. The differences were considered significant when $P<0.05$. The Kaplan-Meier test was used for survival analysis.

\section{RESULTS}

\section{Survival time in dTGR}

Only 5 out of $20(25 \%)$ of untreated dTGRs survived until the age 8 weeks; levosimendan partially prevented Ang II-induced cardiovascular mortality in dTGR (survival rate $11 / 15,73 \%)(P<0.05)$. In the separate survival study, a lifelong levosimendan treatment begun at age 4 weeks increased the mean survival time in dTGR by $58 \%$ (mean survival time in levosimendan-treated dTGR 59.6 \pm 12.6 days, $95 \%$ confidence interval: $34.8-84.3$ days $v$ s. mean survival time in untreated dTGR $37.7 \pm 8.3$ days, $95 \%$ confidence interval: $21.5-53.9$; log-rank test $P=0.004)$. Levosimendan also increased median survival time by 27\% (33 \pm 3.4 days, $95 \%$ confidence interval: 26.4-39.6 days $v s$. $26 \pm 0.7$ days, 95\% confidence interval: 24.5-27.5; log-rank test $P=0.004$ ) (Figure 1).

\section{Cardiac function and cardiac dimensions}

Levosimendan increased EF (Figure 2a) and FS (Figure 2b) both in dTGRs and SD rats. There was no difference in EF or FS between rat strains at the age 8 weeks. Color Doppler imaging showed increased isovolumic relaxation time in dTGRs as compared with $\mathrm{SD}$ rats (26.7 \pm 1.4 vs. $20.3 \pm 2.4 \mathrm{~ms}, P<0.05)$, indicating impaired diastolic relaxation in dTGRs. Levosimendan did not correct Ang II-induced diastolic dysfunction in dTGR (isovolumic relaxation time $27.1 \pm 1.2 \mathrm{~ms}, P>0.05)$. Effects of levosimendan treatment on echocardiographic cardiac dimensions are given in Table 1.

Blood pressure, heart rate, cardiac hypertrophy and levosimendan dosage

Levosimendan did not influence the development of severe hypertension or influence heart rate in dTGRs (Figures $3 \mathrm{a}$ and $\mathrm{b}$ ). In SD rats, levosimendan did not affect blood pressure or heart rate (terminal systolic blood pressure and heart rate in levosimendan-treated SD rats $127 \pm 3 \mathrm{~mm} \mathrm{Hg}$ and $392 \pm 12$ b.p.m., respectively, vs. $132 \pm 3 \mathrm{~mm} \mathrm{Hg}$ and $389 \pm 5$ b.p.m. in SD controls, $P>0.05)$. Levosimendan did not prevent Ang II-induced cardiac hypertrophy or influence cardiac mass in SD rats (Figures $3 \mathrm{c}$ and $\mathrm{d}$ ). The average daily dosage of levosimendan was $1.04 \pm 0.03 \mathrm{mg} \mathrm{kg}^{-1}$ in dTGRs (range: $0.9-1.15$ ) and $1.1 \pm 0.05 \mathrm{mg} \mathrm{kg}^{-1}$ in SD rats (range: $0.73-1.3$ ) (Figure 3e). Terminal plasma levosimendan concentrations in dTGRs were $5.5 \pm 1.2 \mathrm{ng} \mathrm{ml}^{-1}$, and the level of OR-1896, the stable metabolite of levosimendan, was

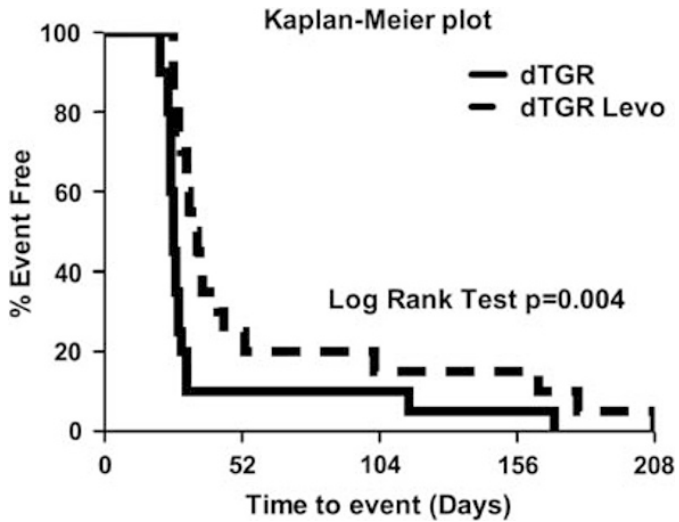

Figure 1 Survival curve of follow-up experimental period until the natural death event of dTGR and dTGR+Levo. The log-rank test was used to compare the Kaplan-Meier survival curves with each other. Levosimendan was administered orally through drinking fluid at the daily dosage of $1 \mathrm{mg} \mathrm{kg}^{-1}$. The dTGR denotes double transgenic rats harboring human renin and angiotensinogen genes, dTGR+Levo-dTGR medicated with levosimendan. Means \pm s.e.m. are given, $n=20$ in each group. Log-rank test $P=0.004$.

$31.7 \pm 5.6 \mathrm{ng} \mathrm{ml}^{-1}$. In $\mathrm{SD}$ rats treated with levosimendan, plasma levosimendan and OR-1896 concentrations averaged $7.6 \pm 3.1$ and $43.4 \pm 5.2 \mathrm{ng} \mathrm{ml}^{-1}$, respectively.

\section{Cardiac morphology and cardiac ANP mRNA expression}

Levosimendan treatment partially prevented Ang II-induced coronary and myocardial damage (Figures $4 \mathrm{a}$ and $\mathrm{b}$ ). Ang II-induced cardiac ANP mRNA overexpression was decreased by $56 \%$ in levosimendantreated dTGRs $(P<0.05)$ (Figure $4 \mathrm{c})$. Representative photomicrographs of cardiac morphology from SD rats and untreated dTGRs are given (Figures $4 \mathrm{~d}$ and e).

\section{Cardiac $\alpha$-MHC, $\beta$-MHC, MCP-1, Bax and PGC- $1 \alpha$ mRNA expressions}

There was no difference between the treatment groups in cardiac $\alpha$-MHC or $\beta$-MHC mRNA expression (Figures $5 \mathrm{a}$ and $\mathrm{b}$ ). Levosimendan produced a non-significant $31 \%$ decrease in $\beta$-MHC in dTGRs, and a $51 \%$ decrease in SD rats (Figure 5b). Cardiac MCP-1 expression was increased five-fold in dTGRs as compared with SD rats (Figure 5c). Levosimendan did not influence Ang II-induced MCP-1 mRNA overexpression. Cardiac Bax mRNA expression was slightly increased in dTGRs as compared with SD rats (Student's $t$-test $P<0.05$ ) (Figure 5d). Levosimendan did not influence cardiac Bax mRNA expression in dTGRs or SD rats. There was no difference between the treatment groups in the cardiac mRNA expression of the mitochondrial biogenesis marker PGC-1 $\alpha$ (Figure 5e).

\section{Cardiac SERCA2a and NCX protein expressions}

Cardiac SERCA2 and NCX protein expressions were lower in dTGR compared with SD rats (Figures 6a and b). Levosimendan did not influence SERCA2 or NCX expression in dTGRs, but increased cardiac SERCA2 in SD rats.

Serum electrolytes, creatinine, liver enzymes and renal morphology Levosimendan did not influence serum biochemical markers in dTGRs or SD rats (Table 2). The renal lesions of dTGRs ranged from a slight thickening of the arteries and tubular dilatation with 


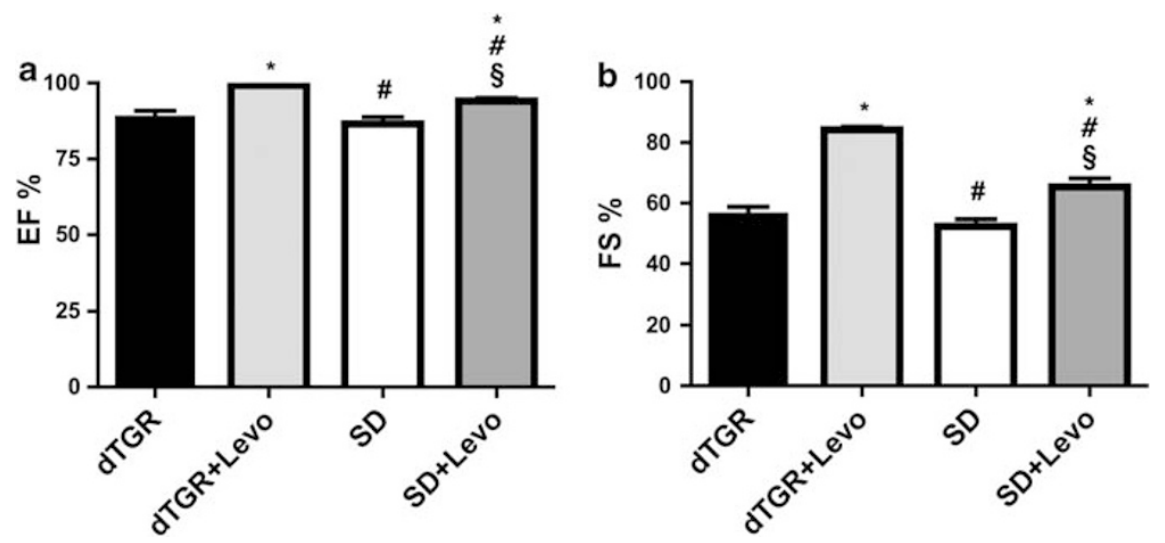

Figure 2 Bar graphs showing the effects of levosimendan 4-week treatments on heart functions (a) EF and (b) FS. Abbreviations: dTGR+Levo denotes dTGR treated with levosimendan; SD+Levo denotes SD rats treated with levosimendan. Means \pm s.e.m. are given, $n=10-14$ in each group. ${ }^{*} P<0.05$ compared with dTGR; ${ }^{\#} P<0.05$ compared with dTGR+Levo; $\$ P<0.05$ compared with SD.

Table 1 Effects of 4-week levosimendan treatment on cardiac function measured by echocardiography in dTGRs and SD rats

\begin{tabular}{|c|c|c|c|c|c|}
\hline Variable & $d T G R(\mathrm{n}=14-15)$ & dTGR+levosimendan $(n=13-14)$ & $S D(\mathrm{n}=10)$ & SD+levosimendan $(\mathrm{n}=10)$ & ANOVA (P-value) \\
\hline LVESD & $3.064 \pm 0.2200$ & $0.970 \pm 0.0796^{*}$ & $3.12 \pm 0.2294$ \# & $2.434 \pm 0.2196 * \# \S$ & $P<0.0001$ \\
\hline LVEDD & $6.936 \pm 0.1293$ & $6.137 \pm 0.2928^{*}$ & $7.108 \pm 0.1410 \#$ & $6.988 \pm 0.1545^{\#}$ & $P=0.0049$ \\
\hline IVS (d), mm & $2.454 \pm 0.07687$ & $2.735 \pm 0.1332$ & $1.836 \pm 0.04840 *$ \# & $1.876 \pm 0.06300 * \#$ & $P<0.0001$ \\
\hline$P W(d), m m$ & $2.389 \pm 0.06099$ & $2.611 \pm 0.1211$ & $1.804 \pm 0.08352 * \#$ & $1.739 \pm 0.03466 *$ \# & $P<0.0001$ \\
\hline $\mathrm{EDV}, \mathrm{ml}$ & $0.7679 \pm 0.03863$ & $0.5779 \pm 0.05838^{*}$ & $0.8180 \pm 0.04572 \#$ & $0.7810 \pm 0.04608 \S$ & $P=0.0039$ \\
\hline ESV, ml & $0.09429 \pm 0.01988$ & $0.002857 \pm 0.00125^{*}$ & $0.1150 \pm 0.02339 \#$ & $0.0460 \pm 0.009214 * \S$ & $P<0.0001$ \\
\hline HR, b.p.m. & $337.5 \pm 5.736$ & $350.0 \pm 7.653$ & $388.6 \pm 4.689 * \#$ & $391.8 \pm 11.78 * \#$ & $P<0.0001$ \\
\hline
\end{tabular}

Abbreviations: dTGR, double transgenic rats harboring human renin-angiotensinogen genes; dTGR+Levo, dTGR treated with levosimendan; HR, heart rate; IVS, interventricular septum; LVEDD, left ventricle end-diastolic dimension; LVESD, left ventricle end-systolic dimension; PW, posterior wall; SD, Sprague-Dawley rats; SD+Levo, SD rats treated with levosimendan. Means \pm s.e.m. are given, $n=10-15$ in each group.

${ }^{*} P<0.05$ vs. dTGR; $\#<<0.05$ vs. dTGR+Levo; ${ }^{\circledR} P<0.05$ vs. SD.
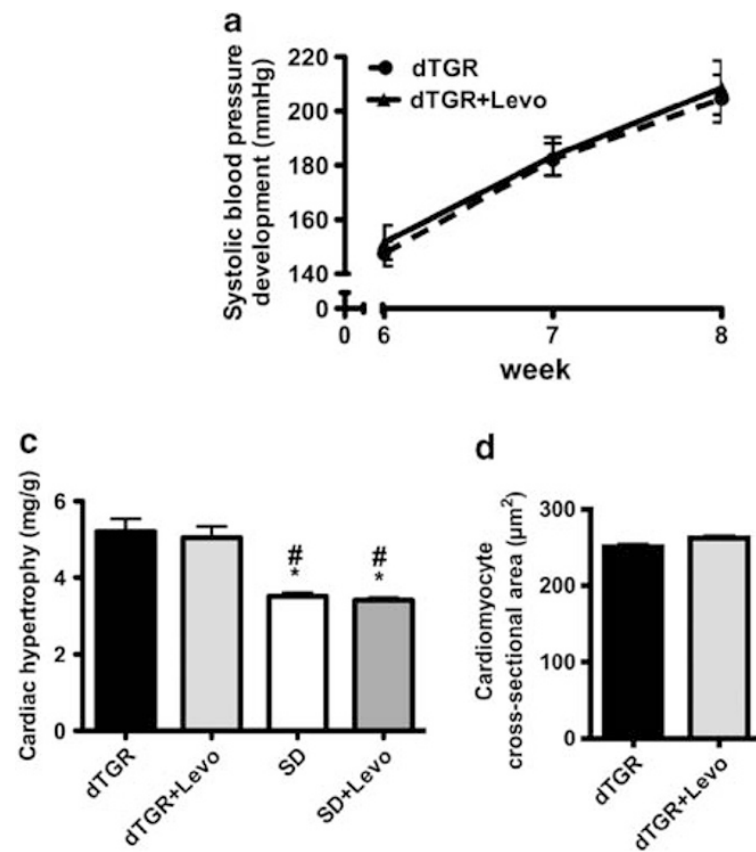

d
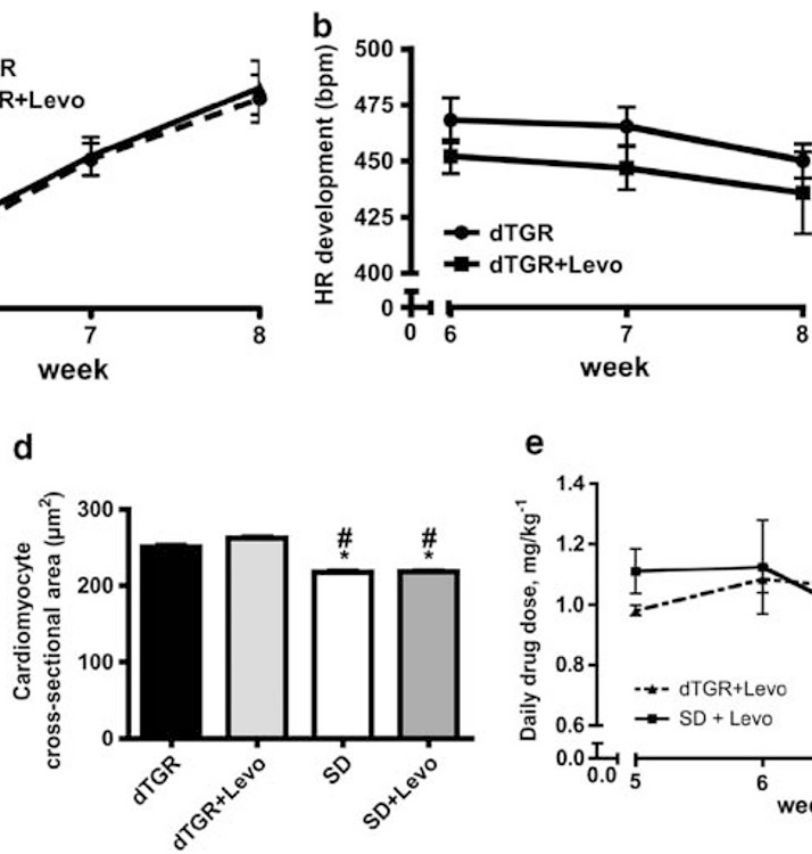

e

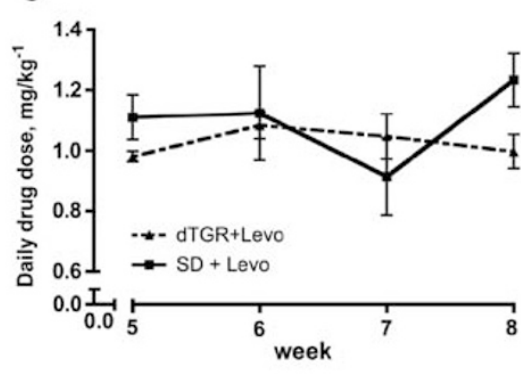

Figure 3 Bar graphs showing the effects of levosimendan 4-week treatments on (a) systolic blood pressure and (b) heart rate development in dTGR and dTGR treated with levosimendan (c) cardiac hypertrophy calculated as heart weight to body weight ratio (d) cardiomyocyte cross-sectional area (e) average weekly levosimendan doses calculated from water consumption during the 4-week experimental period. For abbreviations, see Figure 2. Means \pm s.e.m. are given, $n=10-20$ in each group. ${ }^{*} P<0.05$ compared with dTGR; ${ }^{*} P<0.05$ compared with dTGR+Levo. 

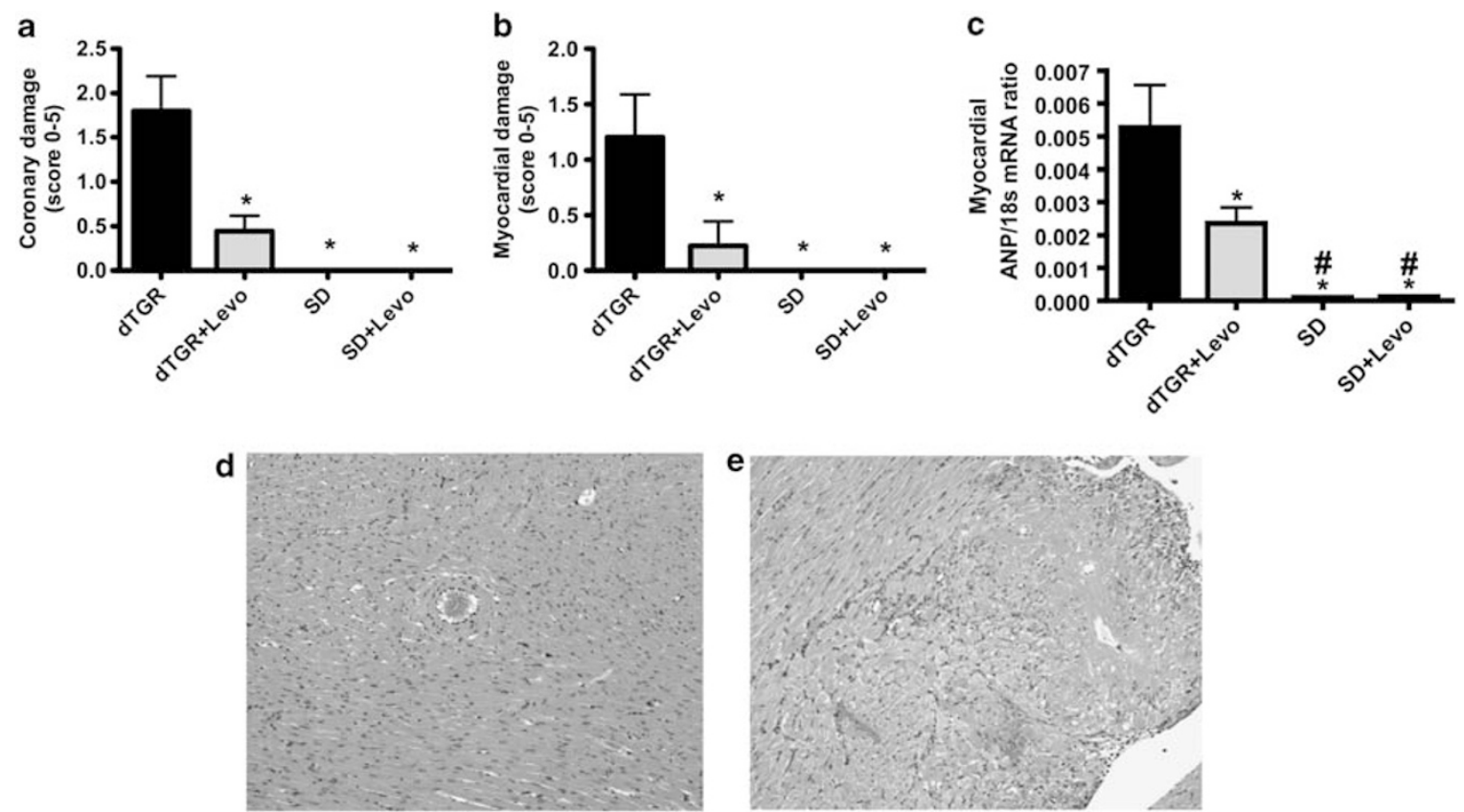

Figure 4 Bar graphs showing the effects of the 4-week levosimendan treatment on (a) coronary damage, (b) myocardial damage, (c) ANP mRNA expression, representative photomicrographs of cardiac morphology from untreated SD (d) and dTGR (e). H\&E staining to 4- $\mu$ m-thick paraffin-embedded sections. Original magnification $\times 200$. For abbreviations, see Figure 2. Means \pm s.e.m. are given, $n=8-10$ in each group. ${ }^{*} P<0.05$ compared with dTGR; ${ }^{\#} P<0.05$ compared with dTGR+Levo.
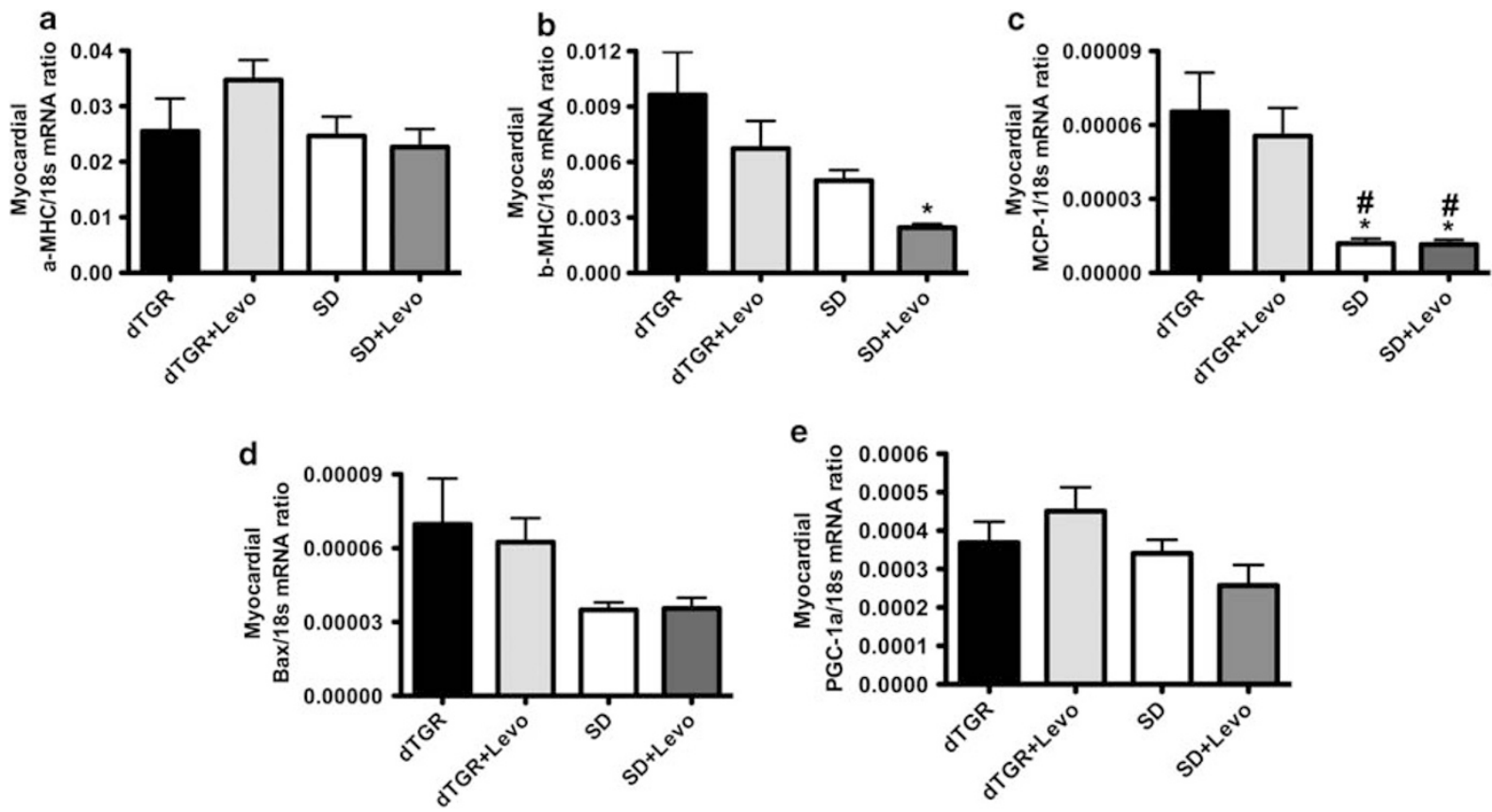

Figure 5 Bar graphs showing the effects of 4-week levosimendan treatment on myocardial mRNA expression of (a) $\alpha-\mathrm{MHC}$, (b) $\beta$-MHC, (c) MCP-1 (d) Bax and (e) PGC-1 $\alpha$. For abbreviations, see Figure 2. Means \pm s.e.m. are given, $n=8-10$ in each group. ${ }^{*} P<0.05$ compared with dTGR; ${ }^{\#} P<0.05$ compared with dTGR+Levo.

proteinaceous casts to diffuse arterial and glomerular necrosis with tubular atrophy/regeneration (Figures $7 \mathrm{a}$ and b). Levosimendan did not significantly influence kidney morphology (Figures 7c-e), albuminuria (Figure 7f) or 24-h urinary excretion rates of sodium, potassium, calcium, phosphorus or creatinine clearance (data not shown).

\section{DISCUSSION}

The use of existing inotropic agents is hampered by poor prognosis due to drug-induced increase in intracellular calcium concentration, induction of cardiac arrhythmias, activation of maladaptative $\mathrm{Ca}^{2+}$ dependent signaling cascades and increase in myocardial oxygen consumption. ${ }^{7}$ We explored whether levosimendan would improve 

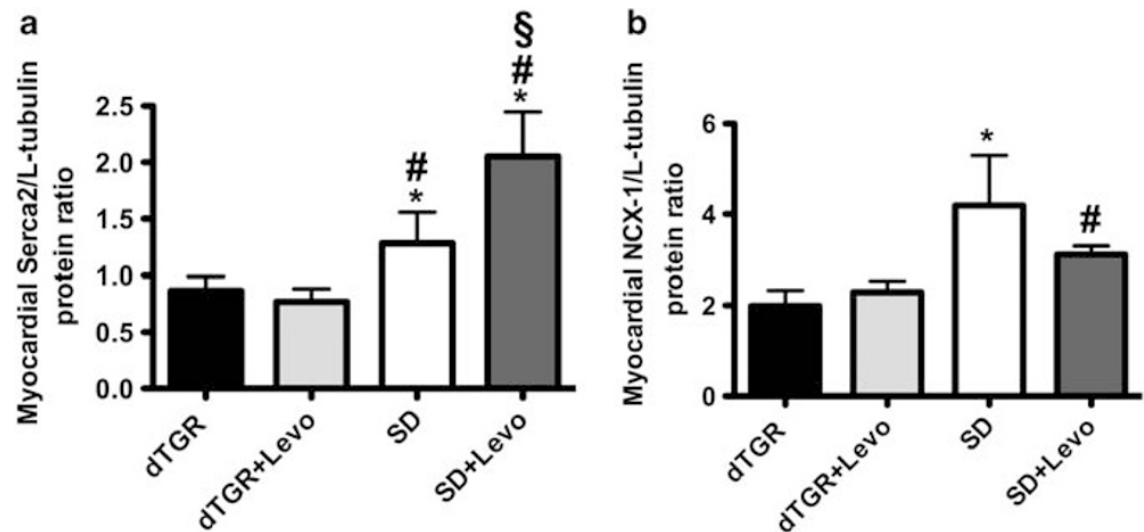

Figure 6 Bar graphs showing the effects of 4-week levosimendan treatment on myocardial protein expression of (a) Serca2 and (b) NCX-1. For abbreviations, see Figure 2. Means \pm s.e.m. are given, $n=8-10$ in each group. ${ }^{*} P<0.05$ compared with dTGR; ${ }^{\#} P<0.05$ compared with dTGR+Levo; ${ }^{\S} P<0.05$ compared to SD.

Table 2 Effects of 4-week levosimendan treatment on serum electrolytes, creatinine and liver enzymes in dTGRs and SD rats

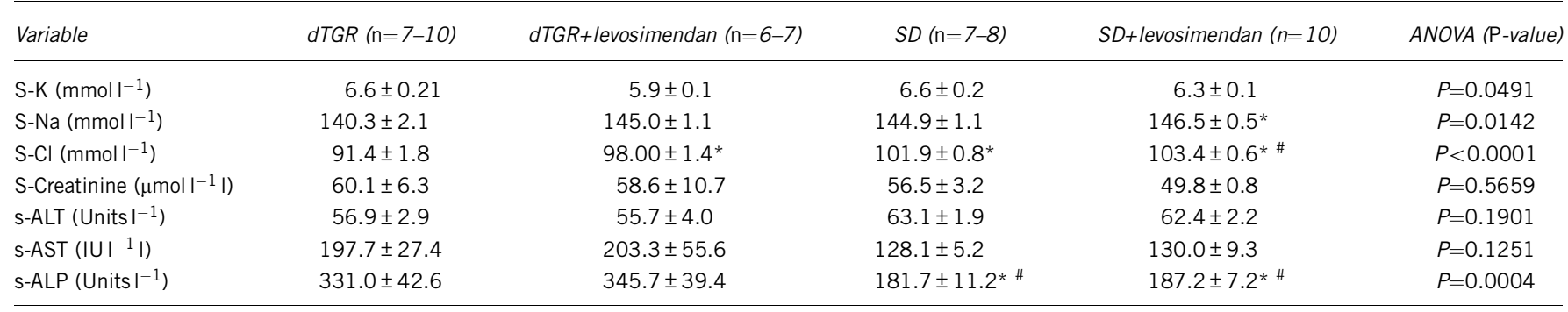

Abbreviations: ALP, alkaline phosphatase; ALT, alanine aminotransferase; AST, aspartate aminotransferase; dTGR, double transgenic rats harboring human renin-angiotensinogen genes; dTGR+Levo, dTGR treated with levosimendan; SD, Sprague-Dawley rats; SD+Levo, SD rats treated with levosimendan.

Means \pm s.e.m. are given, $n=6-10$ in each group.

${ }^{*} P<0.05$ vs. dTGR, $\# P<0.05$ vs, dTGR+Levo.

a

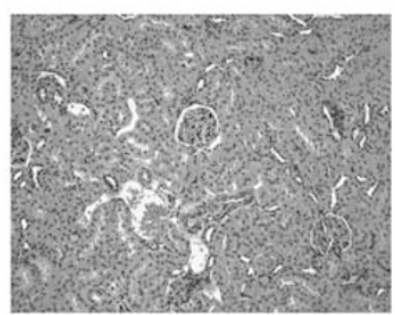

b

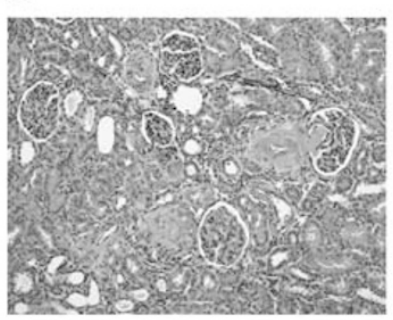

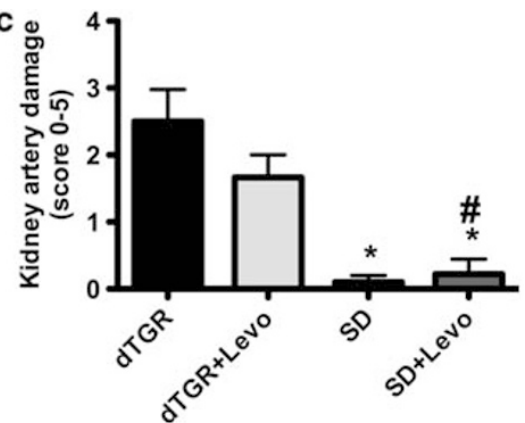

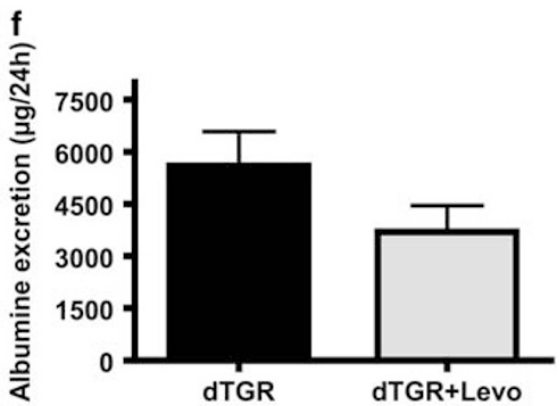

Figure 7 Representative photomicrographs of the renal morphology in (a) SD, (b) dTGR rats and bar graphs showing the effects of 4-week levosimendan treatment on kidney assessed as (c) kidney artery damage, (d) glomerular damage, (e) tubular damage and (f) albumin excretion values. H\&E staining to 4-mm-thick paraffin-embedded sections. Original magnification $\times 200$. Means \pm s.e.m. are given, $n=5-10$ in each group. ${ }^{*} P<0.05$ compared with dTGR; $\# P<0.05$ compared with dTGR+Levo. 
cardiac function and survival in dTGR, a model of Ang II-induced hypertensive heart failure. The important findings in our study were that levosimendan increased mean survival time by almost $60 \%$ and median survival time by $30 \%$ in dTGR. The beneficial effects of levosimendan on life span were blood pressure-independent and were mainly related to improved cardiac function, as measured by increased EF and FS. Levosimendan also ameliorated Ang II-induced cardiac ANP mRNA overexpression and cardiac damage.

Heart failure with preserved systolic function is pathophysiologically characterized by cardiomyocyte hypertrophy and cardiac fibrosis, leading to diastolic stiffness and abnormal relaxation filling of the LV. ${ }^{4}$ Wellner et al. ${ }^{23}$ showed with tissue Doppler measurements that peak early (Ea) to late (Aa) diastolic expansion was reduced in dTGRs, consistent with terminal heart failure from diastolic dysfunction. The animals also showed pronounced cardiomyocyte hypertrophy, preserved systolic function, increased matrix protein expression, downregulation of several genes encoding mitochondrial respiratory chain and lipid metabolism, as well as distinct patterns in the expression profile of genes encoding transcription factors, coagulation, cardiac remodeling, immune system and metabolic pathways. ${ }^{23}$ In very good agreement with Wellner et al.,23 we observed remarkable cardiovascular mortality of dTGRs at the age of 8 weeks and also found diastolic dysfunction by Doppler imaging. In a separate study, we very recently observed distinct patterns of cardiac substrate utilization and mitochondrial dysfunction in dTGRs. ${ }^{27}$

Recently, Koudouna et al. ${ }^{28}$ showed that levosimendan improved the initial outcome of cardiopulmonary resuscitation in an experimental model of cardiac arrest. We found that lifelong levosimendan treatment was associated with a $58 \%$ increase in mean survival time and a $27 \%$ increase in median survival time. Levosimendan did not decrease blood pressure or increase heart rate. These findings suggest that the beneficial effect of levosimendan was largely blood pressure independent. Levosimendan improved systolic ventricular function measured as increased EF and FS through mechanisms likely to be related to calcium sensitization. However, levosimendan did not correct Ang II-induced diastolic dysfunction in dTGRs. Improved cardiac function at systole in levosimendan-treated dTGRs was associated with partial protection against Ang II-induced cardiomyocyte and vascular damage, as well as decreased pressure/volume load imposed to the heart as indicated by the $56 \%$ decrease in cardiac ANP mRNA expression. As the levels of natriuretic peptides correlate with increased end-diastolic pressure and left ventricular wall tension, our findings suggest that levosimendan was able to decrease myocardial tension and the degree of cardiac overload in dTGRs. This effect may have led to improved microcirculation in the heart. In very good agreement with our data, other investigators found that levosimendan and its metabolite OR-1896 elicit a substantial vasodilation and an increase in nitric oxide production in the coronary and skeletal muscle arterioles by activating voltage-sensitive, calcium-activated and ATPsensitive potassium channels. ${ }^{29-31}$ This response to levosimendan could partly be prevented by the K(ATP) channel blockers. ${ }^{29-31}$ Moreover, in an in vivo rat model of coronary ischemia-reperfusion, levosimendan decreased infarct size markedly through the K(ATP) channel-dependent pathway, whereas the PDE-III inhibitor enoximone did not cause any infarct size reduction. ${ }^{32}$ Thus, the role of PDE inhibition as a mediator of the beneficial effects of levosimendan found in this study remains unclear. The lack of any effect of levosimendan on heart rate supports the notion that PDE-III inhibition might have had only a minor role in our animal model. Nonetheless, the levosimendan-induced increase in SERCA2 expression that we observed in our SD rats could suggest an involvement of PDE-III inhibition. Cardiac SERCA2 expression has been shown to be upregulated by increased cellular cAMP levels. ${ }^{33}$ Further studies are thus warranted to examine the influence of PDE-III inhibition, for example, with milrinone treatment, on survival and target-organ damage in this particular animal model.

Ang II triggers inflammatory response and exerts direct proinflammatory effects in part by blood pressure-independent mechanisms related to activation of the redox-sensitive transcription factors nuclear factor- $\kappa \mathrm{B}$ and AP-1, as well as oxidative stress. ${ }^{34-36}$ We showed recently that oral levosimendan ameliorated inflammatory responses, exerted antiapoptotic effects and prevented cardiomyocyte senescence in Dahl/Rapp salt-sensitive rats and in spontaneously diabetic GotoKakizaki rats with postinfarction heart failure. ${ }^{17,18}$ Sareila et al. ${ }^{37}$ recently reported that levosimendan downregulated nuclear factor- $\mathrm{\kappa B}-$ dependent transcription, decreased inducible nitric oxide synthase promoter activity, inducible nitric oxide synthase expression and nitric oxide production using macrophages and fibroblasts exposed to inflammatory stimuli. Therefore, we examined cardiac MCP-1 mRNA expression in our animal model. Surprisingly, levosimendan did not ameliorate Ang II-induced inflammatory response or exert antiapoptotic effects in dTGRs. Although levosimendan is known to also act on mitochondrial ATP-sensitive potassium channels, we did not notice any drug-induced effect on cardiac PGC- $1 \alpha$ mRNA expression, a widely used marker of mitochondrial biogenesis. Moreover, levosimendan was unable to ameliorate Ang II-induced renal damage as assessed by 24 -h albuminuria, creatinine clearance or tissue morphology.

We believe our findings could have clinical relevance. Our study is the first to show that levosimendan can improve cardiac function and survival in a model of Ang II-induced hypertensive heart failure. The fact that the effects were blood pressure independent suggests local pharmacological effects. The combined inotropic and local vasodilatory actions could have resulted in an increased force of contraction, decreased preload and decreased after load in our model. Moreover, by also opening the mitochondrial (ATP)-sensitive potassium channels in cardiomyocytes, the drug could have exerted a cardioprotective and apparently also an anti-inflammatory effect.

\section{CONFLICT OF INTEREST}

The authors declare no conflict of interest.

\section{ACKNOWLEDGEMENTS}

Grants from the Academy of Finland, Päivikki and Sakari Sohlberg Foundation, Foundation of Cardiovascular Research and the Sigrid Jusélius Foundation supported the study. We thank Mrs Anneli von Behr, Ms Nada BecharaHirvonen and Ms Sari Laakkonen for expert technical assistance.

1 del Monte F, Hajjar RJ. Targeting calcium cycling proteins in heart failure through gene transfer. J Physiol 2003; 546: 49-61.

2 Bers DM. Cardiac excitation contraction coupling. Nature 2002; 415: 198-205.

3 Endoh M. Cardiac $\mathrm{Ca}^{2+}$ signaling and $\mathrm{Ca}^{2+}$ sensitizers. Circ J 2008; 72: 1915-1925.

4 Krum H, Abraham WT. Heart failure. Lancet 2009; 373: 941-955.

5 McMurray JJV. Systolic heart failure. N Engl J Med 2010; 362: 228-238.

6 Perrone SV, Kaplinsky EJ. Calcium sensitizer agents: a new class of inotropic agents in the treatment of decompensated heart failure. Int J Cardiol 2005; 103: 248-255.

7 Kass DA, Solaro RJ. Mechanisms and use of calcium-sensitizing agents in the failing heart. Circulation 2006; 113: 305-315.

8 Lee JA, Allen DG. Calcium sensitisers. BMJ 1990; 300: 551-552.

9 Wetzel B, Hauel N. New cardiotonic agents-a promising approach for treatment of heart failure. Trends Pharmacol Sci 1988; 9: 166-170.

10 Hasenfuss G, Pieske B, Castell M, Kretschmann B, Maier LS, Just H. Influence of the novel inotropic agent levosimendan on isometric tension and calcium cycling in failing human heart. Circulation 1998; 98: 2141-2147. 
11 Kota B, Prasad AS, Economides C, Singh BN. Levosimendan and calcium sensitization of the contractile proteins in cardiac muscle: impact on heart failure. J Cardiovasc Pharmacol Ther 2008; 13: 269-278.

12 Lehtonen L, Poder P. The utility of levosimendan in the treatment of heart failure. Ann Med 2007; 39: 2-17.

13 Kleerekoper Q, Putkey JA. Drug binding to cardiac troponin C. J Biol Chem 1999; 274 23932-23939.

14 Kopustinskiene DM, Pollesello P, Saris NE. Levosimendan is a mitochondrial K(ATP) channel opener. Eur J Pharmacol 2001; 428: 311-314.

15 Edes I, Kiss E, Kitada Y, Powers FM, Papp JG, Kranias EG, Solaro RJ. Effects of levosimendan a cardiotonic agent targeted to troponin $C$, on cardiac function and on phosphorylation and $\mathrm{Ca}^{2+}$ sensitivity of cardiac myofibrils and sarcoplasmic reticulum in guinea pig heart. Circ Res 1995; 77: 107-113.

16 Gruhn N, Nielsen-Kudsk JE, Theilgaard S, Bang L, Olesen SP, Aldershvile J. Coronary vasorelaxant effect of levosimendan a new inodilator with calcium-sensitizing properties. J Cardiovasc Pharmacol 1998; 31: 741-749.

17 Louhelainen M, Vahtola E, Kaheinen P, Leskinen H, Merasto S, Kytö V, Finckenberg P, Colucci WS, Levijoki J, Pollesello P, Haikala H, Mervaala EM. Effects of levosimendan on cardiac remodeling and cardiomyocyte apoptosis in hypertensive Dahl/Rapp rats. Br J Pharmacol 2007; 150: 851-861.

18 Louhelainen M, Vahtola E, Forsten H, Merasto S, Kyto V, Finckenberg P, Leskinen H, Kaheinen P, Tikkanen I, Levijoki J, Mervaala E. Oral levosimendan prevents postinfarct heart failure and cardiac remodeling in diabetic Goto-Kakizaki rats. J Hypertens 2009; 27: 2094-2107.

19 Nieminen MS, Cleland JG, Eha J, Belenkov Y, Kivikko M, Poder P, Sarapohja T. Oral levosimendan in patients with severe chronic heart failure-the PERSIST study. Eur $J$ Heart Fail 2008; 10: 1246-1254.

20 Luft FC, Mervaala E, Muller DN, Gross V, Schmidt F, Park JK, Schmitz C, Lippoldt A, Breu V, Dechend R, Dragun D, Schneider W, Ganten D, Haller H. Hypertension-induced end-organ damage: a new transgenic approach to an old problem. Hypertension 1999; 33: 212-218.

21 Mervaala EM, Muller DN, Park JK, Schmidt F, Löhn M, Breu V, Dragun D, Ganten D, Haller H, Luft FC. Monocyte infiltration and adhesion molecules in a rat model of high human renin hypertension. Hypertension 1999; 33: 389-395.

22 Ganten D, Wagner J, Zeh K, Bader M, Michel JB, Paul M, Zimmermann F, Ruf P, Hilgenfeldt U, Ganten U. Species specificity of renin kinetics in transgenic rats harboring the human renin and angiotensinogen genes. Proc Natl Acad Sci USA 1992; 89: 7806-7810.

23 Wellner M, Dechend R, Park JK, Shagdarsuren E, AI-Saadi N, Kirsch T, Gratze P, Schneider W, Meiners S, Fiebeler A, Haller H, Luft FC, Muller DN. Cardiac gene expression profile in rats with terminal heart failure and cachexia. Physiol Genomics 2005; 20: 256-267.

24 Fregly MJ, Wilson KM, Rowland NE, Cade JR. Hyperhydrating effect of acute administration of angiotensin II in rats. Pharmacol Biochem Behav 1992; 41: 183-188.
25 Fregly MJ, Rowland NE. Effect of administration of angiotensin II and isoproterenol, alone and in combination, on drinking and tail skin temperature of the rat. Pharmacol Biochem Behav 1995; 51: 83-88.

26 Herbert RA, Hailey JR, Seely JC, Shackelford CC, Jokinen MP, Wolf JC, Travlos GS. Nomenclature. In: Haschek WM, Rousseaux CG, Wallig MA (eds). Handbook of Toxicologic Pathology. Academic Press: San Diego, 2002. pp 157-167.

27 Mervaala E, Biala A, Merasto S, Lempiäinen J, Mattila I, Martonen E, Eriksson O, Louhelainen M, Finckenberg P, Kaheinen P, Muller DN, Luft FC, Lapatto R, Oresic M. Metabolomics in angiotensin ii induced cardiac hypertrophy. Hypertension 2010; 55: $508-515$.

28 Koudouna E, Xanthos T, Bassiakou E, Goulas S, Lelovas P, Papadimitriou D, Tsirikos N, Papadimitriou L. Levosimendan improves the initial outcome of cardiopulmonary resuscitation in a swine model of cardiac arrest. Acta Anaesthesiol Scand 2007; 51: 1123-1129.

29 Grossini E, Molinari C, Caimmi PP, Uberti F, Vacca G. Levosimendan induces NO production through p38 MAPK, ERK and Akt in porcine coronary endothelial cells: role for mitochondrial K(ATP) channel. Br J Pharmacol 2009; 156: 250-261.

30 Pataricza J, Krassoi I, Hohn J, Kun A, Papp JG. Functional role of potassium channels in the vasodilating mechanism of levosimendan in porcine isolated coronary artery. Cardiovasc Drugs Ther 2003; 17: 115-121.

31 Erdei N, Papp Z, Pollesello P, Edes I, Bagi Z. The levosimendan metabolite OR-1896 elicits vasodilation by activating the K(ATP) and $B K(\mathrm{Ca})$ channels in rat isolated arterioles. Br J Pharmacol 2006; 148: 696-702.

32 Honisch A, Theuring N, Ebner B, Wagner C, Strasser RH, Weinbrenner C. Postconditioning with levosimendan reduces the infarct size involving the PI3K pathway and KATP-channel activation but is independent of PDE-III inhibition. Basic Res Cardiol 2010; 105: 155-167.

33 Hilal-Dandan R, He H, Martin JL, Brunton LL, Dillman WH. Endothelin downregulates SERCA2 gene and protein expression in adult rat ventricular myocytes: regulation by pertussin toxin-sensitive Gi protein and cAMP. Am J Physiol Heart Circ Physiol 2009; 296: H728-H734.

34 Muller DN, Dechend R, Mervaala EM, Park JK, Schmidt F, Fiebeler A, Theuer J, Breu V, Ganten D, Haller H, Luft FC. NF-kappaB inhibition ameliorates angiotensin II-induced inflammatory damage in rats. Hypertension 2000; 35: 193-201.

35 Mervaala EM, Cheng ZJ, Tikkanen I, Lapatto R, Nurminen K, Vapaatalo H, Muller DN, Fiebeler A, Ganten U, Ganten D, Luft FC. Endothelial dysfunction and xanthine oxidoreductase activity in rats with human renin and angiotensinogen genes. Hypertension 2001; 37: 414-418.

36 Muller DN, Shagdarsuren E, Park JK, Dechend R, Mervaala E, Hampich F, Fiebeler A, Ju X, Finckenberg P, Theuer J, Viedt C, Kreuzer J, Heidecke H, Haller H, Zenke M, Luft FC. Immunosuppressive treatment protects against angiotensin II-induced renal damage. Am J Pathol 2002; 161: 1679-1693.

37 Sareila O, Korhonen R, Auvinen $H$, Hämäläinen M, Kankaanranta H, Nissinen E, Moilanen E. Effects of levo- and dextrosimendan on NF-kappaB-mediated transcription iNOS expression and NO production in response to inflammatory stimuli. $\mathrm{Br}$ J Pharmacol 2008; 155: 884-895. 